\title{
At will or not at will: Electrophysiological correlates of preparation for voluntary and instructed task-switching paradigms
}

\author{
Poyu Chen • Shulan Hsieh
}

Published online: 19 December 2014

(C) Psychonomic Society, Inc. 2014

\begin{abstract}
The present study investigated whether the advanced reconfiguration processes of the voluntary switching (VTS) paradigm were different from those of the instructed task switching (ITS) paradigm by examining event-related potentials (ERPs) in a within-subjects design. Of importance, given that effector-to-task mapping might lead to differential preparatory strategies, two effector-to-task mapping groups were studied: the hand-to-task (HAND) and finger-to-task (FINGER) groups. Intriguingly, we found the increased posterior negativity for voluntary switch (and/or increased posterior positivity for voluntary repeat) was exclusive to the HAND group, whereas the increased switch-related late posterior positivity in the ITS paradigm was independent of the effector manipulation. Moreover, the lateralized readiness potentials (LRP) and the mu and beta motor-related amplitude asymmetries indicated that the differential switch-related modulations were not the byproduct of hand-specific preparation. The advanced preparatory strategies in the VTS and ITS paradigms are discussed.
\end{abstract}

Keywords Voluntary task switching $\cdot$ Strategy $\cdot$ Cognitive control $\cdot$ ERP $\cdot$ LRP

\section{Introduction}

Mental flexibility is one of the essential issues in exploring cognitive control. In the previous two decades, the instructed

P. Chen

Department of Psychology, National Chung Cheng University,

Chiayi, Taiwan

e-mail: poyui.chen@gmail.com

S. Hsieh $(\bowtie)$

Department of Psychology, National Cheng Kung University, No. 1, University Road, Tainan 701, Taiwan

e-mail: psyhs1@mail.ncku.edu.tw task switching (ITS) paradigm has been proposed as a persuasive approach to explore mental flexibility (Allport, Styles, \& Hsieh, 1994; Rogers \& Monsell, 1995). ITS requires the participants to switch between cognitive tasks (e.g., parity and magnitude judgments) (for a revew, see Kiesel et al., 2010; Vandierendonck, Liefooghe, \& Verbruggen, 2010). Although studying ITS has provided fruitful evidence for understanding endogenous control, the ITS paradigm appears to be ecologically invalid because real-life situations are not controlled by instructions for imposed tasks. Instead, individuals generate their own decisions to perform tasks sequentially. Recently, Arrington and Logan $(2004,2005)$ proposed a procedure, the voluntary task-switching (VTS) paradigm, in which participants have to select between tasks randomly and equally often at their own will. Given the demanding nature of voluntary selection, Arrington and Logan (2004, 2005) claimed VTS to be a valid tool for exploring endogenous control despite that recent literature has revealed that voluntary task selection was not immune to the stimulusdriven effect (Arrington, 2008; Mayr \& Bell, 2006). To summarize, it has been acknowledged that endogenous control is involved in both VTS and ITS paradigms (Arrington \& Logan, 2005; Rogers \& Monsell, 1995). Nevertheless, it remains unclear whether the underlying preparatory mechanisms of VTS are identical to those of ITS. If there were discrepant preparation processes, we would address which factors might lead to this discrepancy. In the present study, we addressed these issues by incorporating recordings of event-related potentials (ERPs).

As stated in previous ERP literature on ITS (for a review, see Karayanidis et al., 2010), there is an increased late posterior positivity for anticipating a switching task. Some authors have directly related this positive deflection to the P3b component as the construct of "context updating" (Donchin \& Coles, 1988), which suggests that the increased positivity is assumed to reflect the endogenous control for comparison of 
the attributes of incoming stimuli with an internal model and the subsequent revision of the model (Barcelo, MunozCespedes, Pozo, \& Rubia, 2000; Barcelo, Perianez, \& Knight, 2002; Kieffaber \& Hetrick, 2005). Accordingly, the late posterior positivity for switch trials has been interpreted as the advanced endogenous control processes that either update the task sets (Barcelo et al., 2002; Kieffaber \& Hetrick, 2005) and/ or the stimulus-response (S-R) mapping rules (Astle, Jackson, \& Swainson, 2008) in working memory or retrieve the task goals from long-term memory (Jost, Mayr, \& Rosler, 2008). Furthermore, a less common finding in the ITS paradigm is the late frontal negativity ${ }^{1}$ (LFN) for switch trials that occurs in advance of the target presentation (Astle et al., 2008; Lavric, Mizon, \& Monsell, 2008; Mueller, Swainson, \& Jackson, 2007). It remains unclear whether LFN represents the same process as posterior positivity: Lavric et al. (Lavric et al., 2008) suggested that LFN originates from the same dipole as posterior positivity, but Astle et al. (2008) and Mueller et al. (2007) demonstrated that LFN is related to the motor system for preparing new response mappings.

Similarly, there are also event-related components that indicate the advanced reconfiguration in the context of the VTS. Of interest, Vandamme et al. (2010) discovered opposite ERP modulations - that is, an increased contingent negativity variation (CNV)-like deflection over the anterior-posterior scalp for expecting a "voluntary" switch prior to target presentation-as compared to ITS. According to the literature (Van Boxtel \& Böcker, 2004), CNV has been widely connected to reflect response preparation and stimulus anticipation prior to the target presentation, which is in line with the definition of intentional control. Thus, Vandamme et al. (2010) claimed this increased CNV-like activity to be an index of intentional preparation for voluntary switching. In a recent study, Poljac and Yeung (2014) further demonstrated that between-task competition could modulate voluntary intentional control, as revealed by two distinct components in their difficult asymmetric task design (i.e., the easy location task vs. the hard shape task). When additional intentional control was involved in switching to the paradoxically easy task, a right-lateralized frontal CNV was identified to mirror the greater asymmetric switch costs. On the other hand, there was a left-lateralized posterior positivity for switching to the hard task, which indicated the higher retrieval demands of the less familiar task. Interestingly, another recent ERP study directly comparing two paradigms confirmed the opposite ERP modulations in a within-subject design (Kang, Diraddo, Logan, \& Woodman, 2014); that is, they observed the CNV effect in the VTS paradigm but the P3b effect in the ITS paradigm. Specifically, they attributed the $\mathrm{CNV}$ modulations to

\footnotetext{
${ }^{1}$ Differing from the consistent result of the posterior P3b in the ITS paradigm, Jamadar et al. (2010) suggested that the presence of LFN depends on reference recording; those studies with a common mean reference showed LFN, but most studies adopted linked mastoids.
}

an active reconfiguration process in the VTS paradigm but the P3b modulations as memory retrieval in the ITS paradigm.

Although the intuitive argument for the opposite switchrelated modulations between two paradigms was that VTS recruits a rather different reconfiguration process than does ITS based on the fundamental procedure difference (see also Kang et al., 2014), the functional meaning of the increased $\mathrm{CNV}$ amplitude over the anterior and posterior sites in the VTS paradigm is still unclear. Specifically, the coincident characteristics between task switching and hand shifting, i.e., the hand-to-task mapping design in VTS (the digit task is assigned to the left hand, and the letter task is assigned to right hand; Vandamme et al., 2010) raised an alternative explanation: the increased CNV amplitude in the VTS context might reflect effector-related preparation. As proposed by de Jong, Gladwin, and 't Hart (2006), the effector preparatory strategy of switching between response parameters, i.e., hand switching, could be used to ensure proper task control. Moreover, Verleger, Wauschkuhna, van der Lubbea, Jaśkowskia, and Trillenberg (2000) suggested that the fronto-central CNV modulation is related to hand-specific preparation and that the centro-parietal modulation is associated with the S-R rule maintenance. Thus, it seemed that the hand-to-task mapping design provided the participants with a possible effectordependent preparatory strategy for reconfiguring a new task by either adopting a hand-specific preparation or retrieving the S-R rule efficiently based on the distinct hand-to-task link.

Further evidence supporting the effector-dependent preparatory strategy speculation came from our previous study (Chen \& Hsieh, 2013), in which we adopted finger-to-task mapping, i.e., the magnitude task was assigned to the index fingers and the parity task was assigned to the middle fingers, rather than the commonly used hand-to-task mapping. With the spatial characteristics embedded in the digit stimuli, we demonstrated a task choice bias in terms of the S-R correspondence effect that resulted from the overlapping spatial representation between the digit stimulus and the response. Moreover, the general reaction times (RTs) of VTS were inferior to those with ITS, which was not evident in the hand-to-task mapping (Arrington \& Logan, 2005). Taken together, it seemed that participants who did not have the benefit of a distinct hand-to-task association used a rather different preparatory strategy, i.e., the participants kept both tasks available and did not choose which task to perform until the stimulus representation, so that a biased task choice based on the formation of the S-R correspondence effect and lengthened RTs of VTS were observed.

To summarize, it is likely that participants recruit different preparatory strategies depending on the different effector-totask assignments in the VTS context since participants are able to generate diverse preparations based on their own "will" (see also Liefooghe, Demanet, \& Vandierendonck, 2010). The present study sought to clarify whether and how the effector-to-task manipulation elicited different preparatory 
strategies between VTS and ITS paradigms. For better temporal control over the preparation and implementation stages, we adopted an explicit cuing procedure as the ITS, which was similar to Experiment 2 of Arrington and Logan (2005). Accordingly, we directly contrasted VTS with ITS in a within-subjects design, and the effector-to-task mapping (i.e., the hand-to-task (HAND) group vs. the finger-to-task (FINGER) group) was conducted as a between-subjects factor. Given on the shared motor components of VTS and ITS in the same effector-to-task assignments (either the HAND or FINGER group), we could further compare the advanced reconfiguration processes by controlling the hand-specific motor preparation level. Additionally, three cue-target intervals (CTIs) were manipulated as another within-subjects factor.

Various EEG components were measured to dissociate two distinct levels of preparation (Poljac \& Yeung, 2014): non-motorrelated preparation and hand-specific motor preparation (see Table 1). The former level of preparation was targeted to examine whether the effector manipulation elicited different preparatory strategies as reflecting on the CNV activities in the VTS and the late posterior positivity in the ITS environment. Furthermore, a fast-slow ERP analysis was carried out to examine the effectiveness of task preparation (e.g., Lavric et al., 2008; Poljac \& Yeung, 2014). On the other hand, the later level of hand-specific motor preparation, including the lateralized readiness potentials (LRPs) and the frequency band index - the mu $(9-13 \mathrm{~Hz})$ and beta $(18$ $26 \mathrm{~Hz}$ ) movement-related amplitude asymmetry (MRAA) - were used to examine whether the hand-specific motor preparation contributed to the previous observed opposite switch-related modulations between VTS and ITS in the HAND group ${ }^{2}$. By measuring the lateralized motor cortical activities when the two tasks were mapped to different hands, LRP represents the translation process from the task goal to the response execution, and mu- and beta-MRAA indicate motor preparation (de Jong et al., 2006; Poljac \& Yeung, 2014). Accordingly, by comparing LRP and mu- and beta- MRAA of VTS with those of ITS in repeat and switch trials, respectively, we could ensure the involvement of hand-specific preparation in the reconfiguration processes (see also Kang et al., 2014).

\section{Method \\ Participants}

Forty-eight participants ( 26 males), ranging from 18 to 26 years of age (mean age 21.17 years ) were recruited from National Chung Cheng University. All participants had self-

\footnotetext{
$\overline{2}$ The hand-specific preparation indexes are not valid for the FINGER group because no lateralized activities are available until the stimulus representation.
}

reported normal or corrected-to-normal vision and were free of neurological or psychological diseases. Each participant received NT\$500 in compensation for their participation.

\section{Ethical approval}

All subjects provided signed informed consent before the experiments, which was approved by the ethics review committee.

\section{Apparatus and stimuli}

The participants sat comfortably in a sound-attenuated dim room, approximately $70 \mathrm{~cm}$ from the monitor. All target stimuli were centered on the monitor with a black color surrounded by a green or red color patch. The green and red colors were maintained at identical luminance $\left(120 \mathrm{~cd} / \mathrm{cm}^{2}\right)$. The target stimuli were the digits 1 to 9 excluding 5 with a $0.6^{\circ}$ visual angle, and the square color patches were at a $1^{\circ}$ visual angle. The probability of the appearance of red or green patches was equal, whereas the transition of the color patches was maintained at 1:1.

\section{Design and procedure}

The participants were asked to respond to the target stimuli (digits 1 to 9 excluding 5) according to either a magnitude (greater or less than 5) or parity (odd or even) task. One-half of the participants $(n=24)$ were assigned to the HAND group, in which the participants categorized the magnitude task with their left (right) hand while judging the parity task with their right (left) hand. The other half of the participants $(n=24)$ were assigned to the FINGER group and were instructed to use their middle (index) finger for the magnitude task and index (middle) finger for the parity task. All the task polarityto-effector mappings were counterbalanced across subjects. Each participant was provided with two consecutive practice blocks of 64 trials to become familiar with the S-R mapping of the magnitude and parity tasks. After the practice blocks, the participants received ten blocks of 97 trials that included both the VTS and ITS paradigms in a counterbalanced order, i.e., VTS-ITS vs. ITS-VTS order. In each trial, a target was presented after the presentation of one of three randomized cuetarget intervals (CTIs), which were 300,600 , or $900 \mathrm{~ms}$ long, to encourage preparation for the upcoming task. Following the response and a 100-ms interval (Response-Cue interval; RCI), a cue for the next trial appeared. After each block, the participants were given a short break. During the ITS paradigm, the participants were asked to categorize target digits as either the magnitude or parity task according to the color cues. The color-task association was counterbalanced between subjects. During the VTS paradigm, the same green/red color cues served as the warning signal for preparation, and the 
Table 1 EEG components related to non-motor-related preparation and hand-specific motor preparation

\begin{tabular}{|c|c|c|}
\hline Two distinct levels of preparation & Components & Assumptions \\
\hline \multirow{2}{*}{$\begin{array}{l}\text { Non-motor-related preparation: to } \\
\text { examine the effector-dependent } \\
\text { preparatory strategy }\end{array}$} & CNV (VTS) & $\begin{array}{l}\text { H0 (effector-independent preparatory strategy): no different switch-related } \\
\text { modulations between the HAND and FINGER groups }\end{array}$ \\
\hline & $\begin{array}{l}\text { vs. late posterior } \\
\text { positivity/ } \\
\text { P3b (ITS) }\end{array}$ & $\begin{array}{l}\text { H1 (effector-dependent preparatory strategy): different switch-related } \\
\text { modulations between the HAND and FINGER groups }\end{array}$ \\
\hline \multirow[t]{2}{*}{$\begin{array}{l}\text { Hand-specific motor preparation } \\
\text { (HAND group only) }\end{array}$} & $\begin{array}{l}\text { LRP, mu- and } \\
\text { beta-MRAA }\end{array}$ & $\begin{array}{l}\text { H0 (same hand-specific motor preparation between VTS and ITS) : no different modula- } \\
\text { tions between the VTS and ITS paradigms in repeat and switch trials, respectively. }\end{array}$ \\
\hline & & $\begin{array}{l}\text { H1 (different hand-specific motor preparation between VTS and ITS): different modulations } \\
\text { between the VTS and ITS paradigms in repeat and switch trials, respectively. }\end{array}$ \\
\hline
\end{tabular}

$C N V$ contingent negativity variation, VTS voluntary task switching, ITS instructed task switching, LRP lateralized readiness potentials, MRAA movement-related amplitude asymmetry

participants were explicitly informed that the cues were meaningless. Of importance, participants were requested to perform a task as randomly and equally as possible (see also Experiment 2, Arrington and Logan, 2005).

\section{EEG acquisition and formation}

EEG was continuously recorded by a 32-channel Q-cap (Agcl32 electrocap; Neuroscan, Inc. El Paso, TX, USA). Two electrodes were placed above or below the left eye to record vertical EOGs, whereas two electrodes were attached on the outer canthi of each eye to record horizontal EOGs. A ground electrode was placed on the forehead. All electrodes were initially referenced online to the left mastoid and offline to the average of the left and right mastoids. All electrode impedances were reduced below 5 $\mathrm{k} \Omega$. The EEG and EOG signals were continuously sampled at $250 \mathrm{~Hz}$ on a Synamps 1 amplifier with an online band-pass of $0.1-30 \mathrm{~Hz}$. The vertical eyeblink artifact was corrected in the continuous EEG by an ocular reduction algorithm offered by the Neuroscan software. These segments were further rejected if the signals' drift exceeded $\pm 50 \mu \mathrm{V}$ from baseline according to the Neuroscan software or if the A/D converter became saturated with visual inspections. The total rejection rate across the different conditions was approximately $9.74 \%$.

To observe the overall dynamic EEG activities from cue onset to the subsequent target implementation, cue-locked epochs were constructed from $100 \mathrm{~ms}$ before the cue onset to the end $300 \mathrm{~ms}$ of the target. An average mean amplitude between the -50 and $50 \mathrm{~ms}$ baseline (Karayanidis, Coltheart, Michie, \& Murphy, 2003) was employed. A baseline correction was performed for each condition separately.

Regarding the hand-specific motor preparation, the LRP and mu- and beta-MRAA were included. Preparatory mu- and beta-MRAA were quantified for each trial by band-pass filtering the EEG epochs from $\mathrm{C} 3$ and $\mathrm{C} 4$ channels. After filtering, the mu and beta oscillations were quantified by the Hilbert transform ("Hilbert" function in Matlab) for discarding the phase information and acquiring oscillatory power over time
(Poljac \& Yeung, 2014). The time windows of interest for observing the hand-specific preparation were time-locked to the cue onset, retrieved from $100 \mathrm{~ms}$ before the cue onset to the last $1000 \mathrm{~ms}$ of the target, for exploring the foreperiod hand-specific preparation ${ }^{3}$. To obtain LRPs and mu- and betaMRAA, the average amplitudes at the $\mathrm{C} 3$ electrode and $\mathrm{C} 4$ were computed as follows: right-hand (C3-C4)-left-hand (C3-C4) (Coles, 1989).

\section{Data analysis}

After removing the error trials, the following trials were also excluded from the behavioral and neurophysiological analyses: trials with RTs less than $150 \mathrm{~ms}$ or greater than 3,000 ms, the initial trials of each block, and the trials following the error trials.

To observe the dynamic task preparatory processes, we examined the cue-locked ERPs in three CTI levels, separately. After visual inspection, the mean amplitude of cue-locked ERP modulations and topographic maps were performed in 300 -ms time windows, e.g., a time window of $0-300 \mathrm{~ms}$ in CTI $300 \mathrm{~ms}$, time windows of $0-300 \mathrm{~ms}$ and $300-600 \mathrm{~ms}$ in CTI $600 \mathrm{~ms}$, and time windows of 0-300 ms, 300-600 ms, and 600-900 ms in CTI $900 \mathrm{~ms}$, respectively.

For the hand-specific motor preparation analysis in the HAND groups, the continuous paired t-tests of the LRPs and the mu- and beta-MRAA were conducted to contrast VTS with ITS paradigms in repeat and switch trials, respectively, during the CTI intervals. Only ten significant (not significant) consecutive sample points were regarded as the onset (offset) of a significantly different period (Astle et al., 2008).

\footnotetext{
${ }^{3}$ Data from the first $500 \mathrm{~ms}$ and the last $300 \mathrm{~ms}$ of each timeframe of interest ( $-100 \mathrm{~ms}$ to $1000 \mathrm{~ms}$ after target onset) were discarded to avoid contamination from edge artifacts after filtering in the mu- and betaMRAA data processing [see also Poljac \& Yeung, 2014].
} 


\section{Results}

It must be noted that the current design with a $1: 1$ cue to task mapping would lead the cue transition to always coincide with the task transition in the ITS paradigm. However, the task transition was independent of the cue transition effect in the VTS paradigm. We treated the cue transition as an additional factor to examine whether the cue transition effect contributed to the task transition effect in the VTS context. Although participants were more likely to switch task in the cue switch condition as compared to the cue repetition condition, the ERP data did not reveal the cue transition effect. To simplify and generate an equal comparison between the VTS and ITS paradigms, we only adopted those VTS trials with "corresponding" cue-task transitions, which were the VTS repeat with cue repeat (cueRep/ VTSrep) and VTS switch with cue switch (cueSwt/VTSswt) trials in the following behavioral and neurophysiological data. Furthermore, with regard to the possible carryover effects of the paradigm order (i.e., VTS-ITS vs. ITS-VTS), we treated the order effect as another between-subjects factor. Because the order effect did not interact with the main notable factors in our analysis, we did not address this order effect problem.

\section{Behavioral data}

\section{Choice data: the probabilities of task repetition (VTS only)}

Table 2 indicates the probabilities of task repetition that the participants chose as a function of CTI separated for two mapping groups. We conducted a mixed ANOVA with one between-subjects factor (mapping group: HAND vs. FINGER) and one within-subjects factor (CTI: 300, 600, and $900 \mathrm{~ms}$ ). The results demonstrated that neither the main effect of mapping, $F(1,46)=.93, p=.34$, nor the interaction between mapping and CTI, $F(2,92)=1.66, p=.20$, were significant. Only the CTI main effect was significant, $F(2,92)=7.67$, $p<.05$. A post hoc analysis showed that the repetition bias on CTI $300 \mathrm{~ms}(0.57)$ was significantly greater than the repetition bias on CTI $900 \mathrm{~ms}(0.54), p<.05$.

Although there was no mapping group main effect, a planned comparison was conducted for testing whether the participants with different effector-to-task assignments showed different preparatory strategies. By comparing the overall

Table 2 The probabilities of task repetition as a function of cue-target intervals (CTIs)

\begin{tabular}{lllll}
\hline P(repetition) & 300 & 600 & 900 & Overall \\
\hline HAND & 0.59 & 0.57 & 0.55 & 0.57 \\
SE & 0.02 & 0.02 & 0.02 & 0.02 \\
FINGER & 0.55 & 0.54 & 0.53 & 0.54 \\
SE & 0.02 & 0.02 & 0.02 & 0.02 \\
\hline
\end{tabular}

repetition rate with 0.5 in the HAND and FINGER group, respectively, there was a robust repetition bias in the HAND group $(0.57)(t(1,23)=3.23, p<.01)$ but not in the FINGER group $(0.54)(t(1,23)=3.23, p=.07)$.

\section{Reaction times and proportion of errors}

Figure 1 depicts the mean RTs and proportion of errors (PEs) for the two mapping groups separately. A four-way ANOVA of RT (Table 3) and PE with one between-subjects factor (mapping group) and three within-subjects factors (CTI, paradigm, and transition) allowed us to verify whether advance preparation could eliminate the switch cost in the VTS and ITS paradigms across the two mapping groups.

It is important to note that we generally replicated previous findings (Arrington \& Logan, 2005), i.e., the switch cost was larger in the ITS $(195.89 \mathrm{~ms})$ than in the VTS $(122.10 \mathrm{~ms})$ paradigm, and the switch cost decreased significantly with increased CTI (switch cost means $=185.69,157.16$, and $134.14 \mathrm{~ms}$ for CTI 300, 600, and $900 \mathrm{~ms}$, respectively). In addition, we demonstrated that the CTI effect was more salient in the VTS, $F(2,184)=82.01(\mathrm{RTs}=878.43,822.86$, and $797.46 \mathrm{~ms}$ for CTI 300,600 , and $900 \mathrm{~ms}$, respectively), than the ITS paradigm, $F(2,184)=26.33(\mathrm{RTs}=787.02,755.52$, and $741.14 \mathrm{~ms}$ for CTI 300, 600, and $900 \mathrm{~ms}$, respectively).

With regard to whether the effector manipulation led to differential strategies, an interaction between mapping and transition was found; in this case, the switch cost was greater in the HAND group $(183.85 \mathrm{~ms})$ than in the FINGER group (134.14 ms). Although there was no significant four-way interaction, we carried out planned three-way ANOVAs (paradigm $\times$ CTI $\times$ transition) in the HAND and FINGER groups, respectively, to clarify the effector-dependent strategy. Interestingly, the following planned comparison showed that the paradigm effect was only evident in the FINGER group ( $F(1$, $23)=7.27, p<.05$ ), indicating an overall performance lengthening for the VTS paradigm as compared to the ITS paradigm. This finding further confirmed previous results (Chen \& Hsieh, 2013) and implicated a different preparatory strategy in the FINGER group as compared to the HAND group.

For the PE data, the participants committed more errors in the VTS paradigm than in the ITS paradigm, $F(1,46)=8.84$, $p<.01$. Moreover, the PE for the switch trials were larger than for the repeat trials, $F(1,46)=15.18, p<.01$. A significant twoway interaction between CTI and transition was observed, $F(2,92)=4.23, p<.05$, indicating a significant preparatory effect for PE switch costs.

The ERPs index of task preparation: cue-locked ERP analysis

Figure 2 separately depicts the cue-locked ERP waveforms for the three CTI levels at the three midline electrode sites, Fz, Cz, and $\mathrm{Pz}$, for the repeat and switch trials in the VTS and ITS 


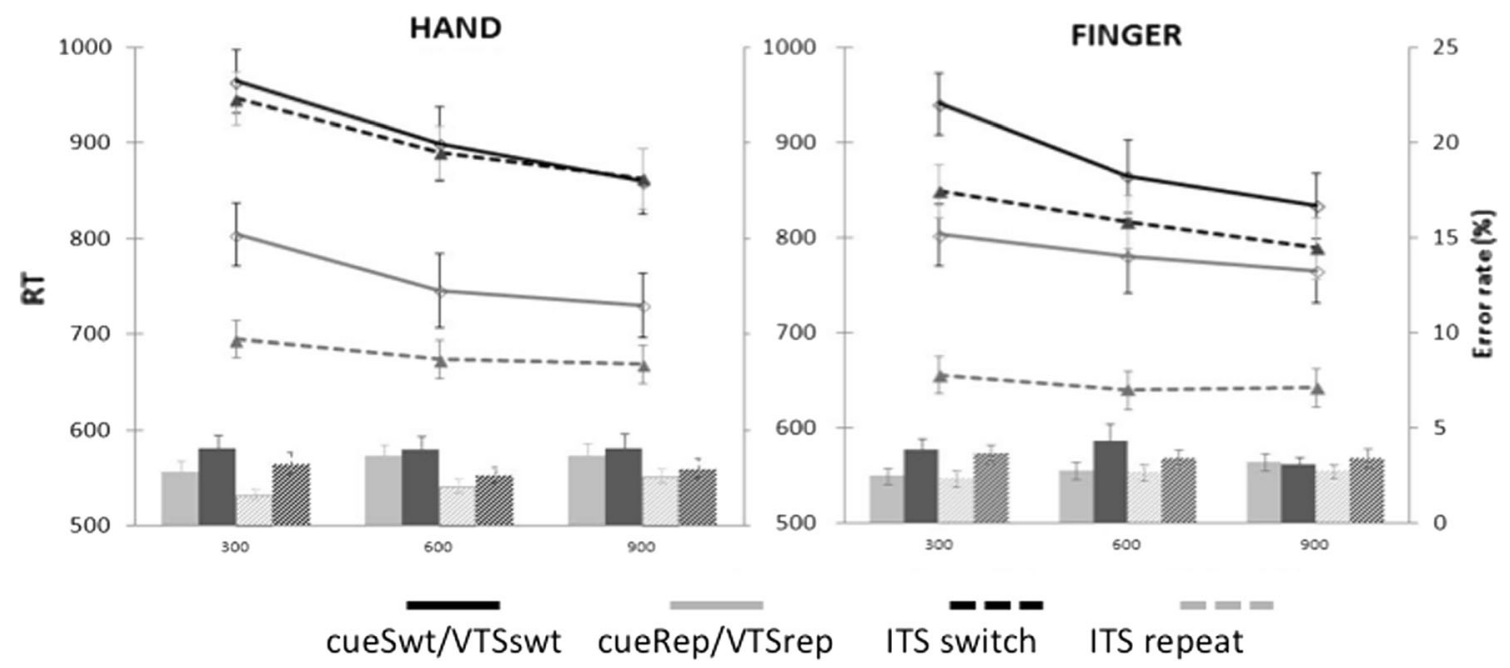

Fig. 1 The behavioral results. The mean reaction times (RTs) $( \pm$ S.E.) and proportion of errors (PEs) ( \pm S.E.) as a function of cue-target interval (CTI) between the voluntary task switching (VTS) (solid lines) and

paradigms by the two mapping groups. The topographic maps of the transition effects are depicted for the VTS and ITS paradigms based on the different time windows in the HAND and FINGER groups. In contrast to the clear CNV activities in previous VTS studies (Poljac \& Yeung, 2014; Vandamme et al., 2010), the present study revealed a rather positivegoing deflection after the 300-ms cue onset in the VTS paradigm.

The mean amplitudes for each time window were subjected to four-way ANOVAs with one between-subjects factor (mapping groups) and three within-subjects factors (site, paradigm, and transition). The overall results of the ANOVAs regarding the

Table 3 Outcome of ANOVAs conducted on reaction times (RTs)

\begin{tabular}{lcc}
\hline Source & F & $\eta^{2}$ \\
\hline Mapping & 1.05 & 0.03 \\
CTI & $81.34^{*}$ & 0.64 \\
CTI $\times$ mapping & 1.61 & 0.03 \\
Paradigm & $6.40^{*}$ & 0.12 \\
Paradigm $\times$ mapping & 0.92 & 0.02 \\
Transition & $386.77^{*}$ & 0.89 \\
Transition $\times$ mapping & $9.45^{*}$ & 0.17 \\
CTI $\times$ paradigm & $10.10^{*}$ & 0.18 \\
CTI $\times$ paradigm $\times$ mapping & 0.01 & 0.00 \\
CTI $\times$ transition & $20.48^{*}$ & 0.31 \\
CTI $\times$ transition $\times$ mapping & 0.51 & 0.01 \\
Paradigm $\times$ transition & $12.04^{*}$ & 0.21 \\
Paradigm $\times$ transition $\times$ mapping & 0.01 & 0.00 \\
CTI $\times$ paradigm $\times$ transition & 0.09 & 0.00 \\
CTI $\times$ paradigm $\times$ transition $\times$ mapping & 2.48 & 0.05 \\
\hline
\end{tabular}

CTI cue-target interval. ${ }^{*} p<.05$ instructed task switching (ITS) (dotted lines) paradigms for task repetition (grey) and switches (black), as separated by the HAND (left panel) and FINGER (right panel) groups

separated time windows in three CTI levels are presented in Table 4. All the analyses were corrected for sphericity violations with Greenhouse-Geisser adjustments. In the following section, we focus on the main interactions of interest in the separate time windows for the three CTIs. In the final section, we present the fast-slow analysis to further reveal the effectiveness index of preparation.

\section{Early stage (0-300 ms)}

With regard to the effector-related effect on the ERP modulations, it was evident that the overall waveforms of the HAND group at $\mathrm{Pz}$ were more negative than those of the FINGER group, $F(1,138)=3.24,3.14$, and 3.87 in CTI 300-, 600-, and 900 -ms conditions, respectively, all $p_{s}<.05$, but not at either $\mathrm{Fz}$ or $\mathrm{Cz}$. Thus, it seemed that the increased negativities of the HAND group compared to the FINGER group were not just the byproduct of the between-subjects factor given on the null effector effect at both $\mathrm{Fz}$ and $\mathrm{Cz}$.

\section{Middle stages (300-600 ms)}

Following the effector-related modulations of the early stage, the increased negativities of the HAND group compared to the

Fig. 2 The cue-locked event-related potential (ERP) waveforms and brain topographies on three cue-target interval (CTI) conditions. The cue-locked waveforms are depicted for the repeat and switch trials in the voluntary switching (VTS) and instructed task switching (ITS) paradigms in the three CTIs and separated by the effector manipulations. For both the HAND and FINGER groups, the topographic maps separately represent the cue-locked transition main effects in different time ranges for the VTS and ITS paradigms, respectively. The vertical grey dashed line indicates the target onset 


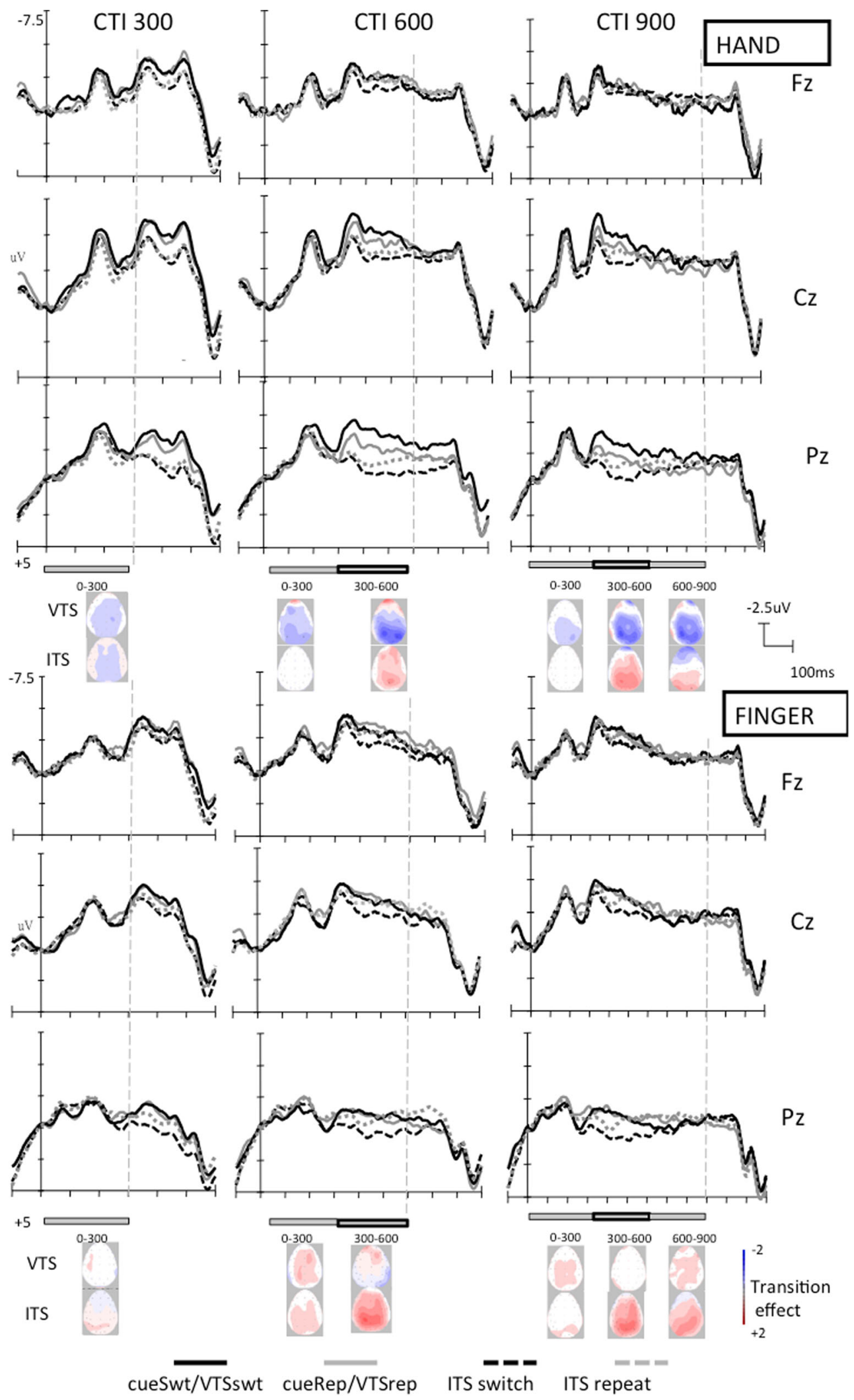

FINGER group sustained in the middle stage for only $\mathrm{Pz}(F(1$, respectively, all $p \mathrm{~s}<.05$ ) but not for either $\mathrm{Fz}$ or $\mathrm{Cz}$. In addition 138) $=4.72$ and 3.76 in CTI 600- and 900-ms conditions, to the effector-related effect, there was also a paradigm 
Table 4 Outcome of ANOVAs conducted on mean amplitudes in the successive time windows in the three cue-target interval (CTI) levels

\begin{tabular}{|c|c|c|c|c|c|c|c|c|c|c|c|c|c|}
\hline \multirow{3}{*}{ Factors } & \multirow[b]{3}{*}{ df } & \multirow{2}{*}{\multicolumn{2}{|c|}{$\frac{\text { CTI300 }}{0-300}$}} & \multicolumn{4}{|l|}{ CTI600 } & \multicolumn{6}{|l|}{ CTI900 } \\
\hline & & & & \multicolumn{2}{|l|}{$0-300$} & \multicolumn{2}{|l|}{$300-600$} & \multicolumn{2}{|l|}{$0-300$} & \multicolumn{2}{|l|}{$300-600$} & \multicolumn{2}{|l|}{$600-900$} \\
\hline & & $\mathrm{F}$ & $\eta^{2}$ & $\mathrm{~F}$ & $\eta^{2}$ & $\mathrm{~F}$ & $\eta^{2}$ & $\mathrm{~F}$ & $\eta^{2}$ & $\mathrm{~F}$ & $\eta^{2}$ & $\mathrm{~F}^{2}$ & $\eta^{2}$ \\
\hline Mapping & 1,46 & 0.24 & 0.01 & 0.13 & 0.00 & 0.86 & 0.02 & 0.07 & 0.00 & 0.37 & 0.01 & 1.18 & 0.03 \\
\hline Site & 2,92 & $6.12 *$ & 0.12 & $13.33^{*}$ & 0.23 & $36.91 *$ & 0.45 & $14.43 *$ & 0.24 & $33.58^{*}$ & 0.42 & $18.90 *$ & 0.29 \\
\hline Site $\times$ mapping & 2,92 & $3.79 *$ & 0.08 & $5.74 *$ & 0.11 & $6.41^{*}$ & 0.12 & $6.60^{*}$ & 0.13 & $5.05^{*}$ & 0.10 & 2.09 & 0.04 \\
\hline Paradigm & 1,46 & 1.42 & 0.03 & 0.04 & 0.00 & $13.79 *$ & 0.23 & 0.22 & 0.01 & $10.34 *$ & 0.18 & 0.02 & 0.00 \\
\hline Paradigm $\times$ mapping & 1,46 & 3.30 & 0.07 & 0.14 & 0.00 & 1.25 & 0.03 & 0.04 & 0.00 & 0.11 & 0.00 & 0.17 & 0.00 \\
\hline Transition & 1,46 & 1.27 & 0.03 & 0.97 & 0.02 & 3.78 & 0.08 & 0.51 & 0.01 & $4.2 *$ & 0.08 & 0.09 & 0.18 \\
\hline Transition $\times$ mapping & 1,46 & 3.49 & 0.07 & $8.31 *$ & 0.15 & 2.91 & 0.06 & 2.96 & 0.06 & 1.78 & 0.04 & $9.92 *$ & 0.01 \\
\hline Site $\times$ paradigm & 2,92 & $5.08^{*}$ & 0.10 & 0.87 & 0.02 & $6.59 *$ & 0.13 & 0.90 & 0.02 & $5.47 *$ & 0.11 & 0.51 & 0.05 \\
\hline Site $\times$ paradigm $\times$ mapping & 2,92 & $8.92 *$ & 0.16 & 2.23 & 0.05 & $8.89 *$ & 0.16 & 0.48 & 0.01 & 1.09 & 0.02 & 2.30 & 0.00 \\
\hline Site $\times$ transition & 2,92 & 1.32 & 0.03 & 0.04 & 0.00 & 0.29 & 0.01 & 0.09 & 0.00 & $3.37 *$ & 0.07 & 0.01 & 0.00 \\
\hline Site $\times$ transition $\times$ mapping & 2,92 & $4.15^{*}$ & 0.08 & 1.69 & 0.04 & 0.89 & 0.02 & $8.94 *$ & 0.16 & 2.33 & 0.05 & $3.33 *$ & 0.07 \\
\hline Paradigm $\times$ transition & 1,46 & 1.45 & 0.03 & 1.81 & 0.04 & $19.77^{*}$ & 0.30 & 0.04 & 0.00 & $21.09^{*}$ & 0.31 & 2.71 & 0.06 \\
\hline Paradigm $\times$ transition $\times$ mapping & 1,46 & 1.96 & 0.04 & $5.72 *$ & 0.11 & 3.25 & 0.07 & 2.88 & 0.06 & 2.03 & 0.04 & 0.89 & 0.02 \\
\hline Site $\times$ paradigm $\times$ transition & 2,92 & 0.54 & 0.01 & 1.02 & 0.02 & $17.98^{*}$ & 0.28 & 2.30 & 0.05 & $21.23 *$ & 0.32 & $18.32 *$ & 0.29 \\
\hline Site $\times$ paradigm $\times$ transition $\times$ mapping & 2,92 & 2.56 & 0.05 & 0.01 & 0.00 & $4.53^{*}$ & 0.09 & 2.82 & 0.06 & $9.57^{*}$ & 0.17 & $7.98^{*}$ & 0.15 \\
\hline
\end{tabular}

${ }^{*} p<.05$

difference over the centro-parietal sites. As revealed in Fig. 2, the VTS paradigm showed more consistently negative activities than did the ITS paradigm at both $\mathrm{Cz}(F(1,138)=9.7$ and 7.48 in CTI 600- and 900-ms, respectively, all $p \mathrm{~s}<.05$ ) and $\mathrm{Pz}$ $(F(1,138)=23.29$ and 7.84 in CTI $600-$ and $900-m s$ conditions, respectively, all $p \mathbf{s}<.05$ ) but not at Fz.

Critically, the significant four-way interaction revealed that the effector manipulation further modulated the transition effect between the VTS and ITS paradigms. The following simple interaction effects in the HAND group $(F(2,92)=20.06$ and 29.57 in CTI 600 and $900 \mathrm{~ms}$, respectively, all $p$ s $<.01$ ) replicated previous findings (Kang et al., 2014) on the reversed polarity transition effect between VTS and ITS; the mean amplitude was more negative for the switch trials than for the repeat trials in the VTS paradigm (for Pz, $F(1,276)=27.22$ and 17.52 in CTI 600and $900-\mathrm{ms}$ conditions, respectively, $p s<.01$; and for $\mathrm{Cz}, F(1$, 276) $=9.99$ in CTI $900 \mathrm{~ms}$ ), whereas the mean amplitude was more positive for the switch trials than for the repeat trials in the ITS paradigm (for $\mathrm{Pz}, F(1,276)=21.43$ and 28.64 in CTI 600and $900 \mathrm{~ms}$, respectively, $p \mathrm{~s}<.01$; for $\mathrm{Cz}, F(1,276)=8.39, p<.01$, in CTI $900 \mathrm{~ms})$. As for the FINGER group, no transition effect was found in the VTS paradigm, whereas the switch-related positivities were replicated in the ITS paradigm at both $\mathrm{Cz}(F(1,138)=5.65$ and 7.9 in CTI 600- and 900-ms conditions, respectively, $p \mathrm{~s}<.05)$ and $\mathrm{Pz}$ $(F(1,138)=12.99$ and 18.93 in CTI 600- and 900-ms conditions, respectively, $p \mathbf{s}<.05)$.

\section{Late stage (600-900 ms)}

As Table 4 demonstrates, there was a significant four-way interaction; the following simple interaction effect tests in the HAND group indicated that there were more negative amplitudes for the switch trials than for the repeat trials in the VTS paradigm at $\mathrm{Pz}(F(1,276)=24.85, p<.001)$, whereas no switch-related modulations were observed for the ITS paradigm in this time window $(F(1,276)=2.51, p=.12)$. As for the FINGER group, there was only a main effect of site $(F(2,46)=6.80, p<.01)$. No other main effects or interactions were observed.

In summary, the critical aspect of the effector manipulation showed that the differential switch-related modulations was exclusive to the VTS paradigm; participants in the HAND group showed increased negativities for the switch trials compared to the repeat trials, whereas no such switch-related modulations were identified in the FINGER group. On the contrary, there were consistent posterior switch-related positivities approximately $300 \sim 600 \mathrm{~ms}$ after cue onset in the ITS paradigm regardless of the effector manipulation (Mueller et al., 2007). Furthermore, it was noteworthy that the amplitude of the HAND group was more negative than the amplitude of the FINGER group, especially at the posterior site, from the beginning to the end of the preparatory intervals. 

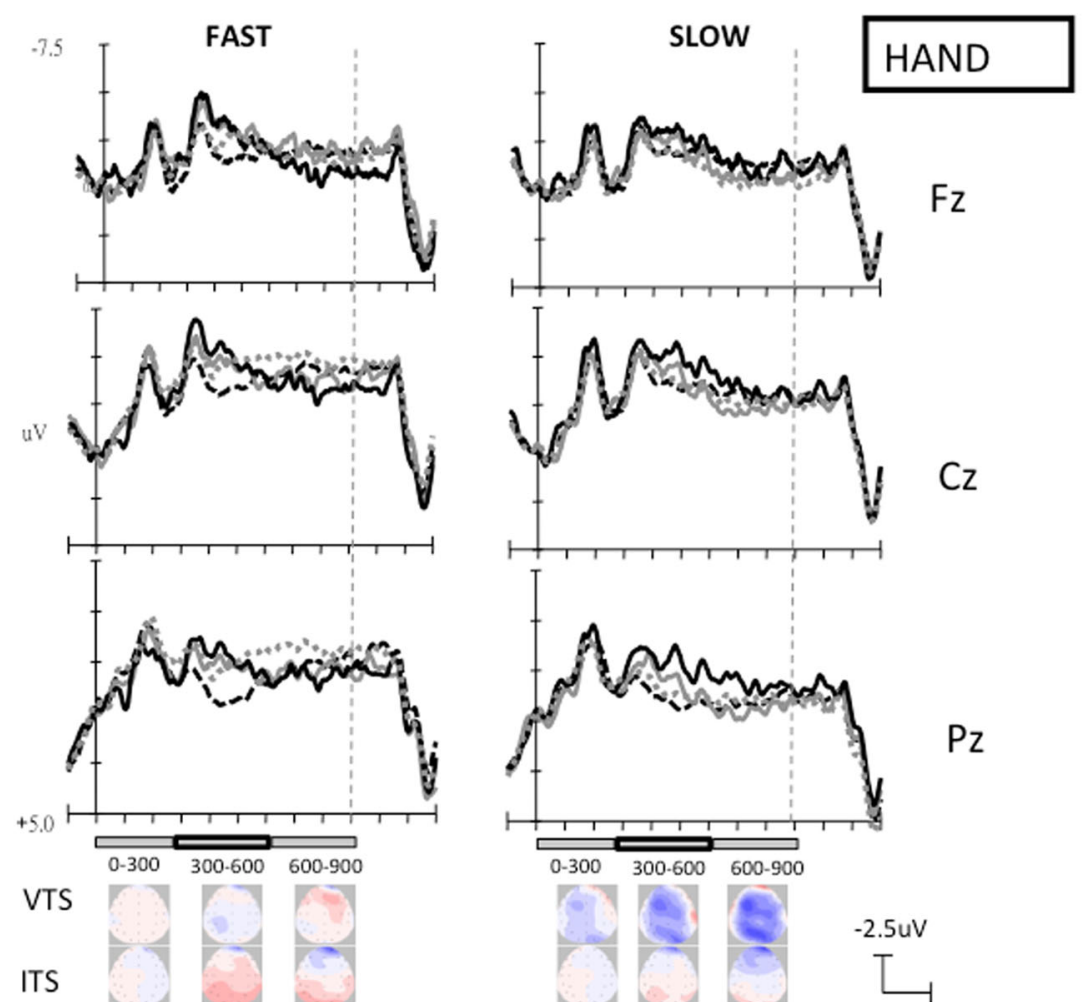

\section{$\mathrm{Cz}$}

$\mathrm{Pz}$
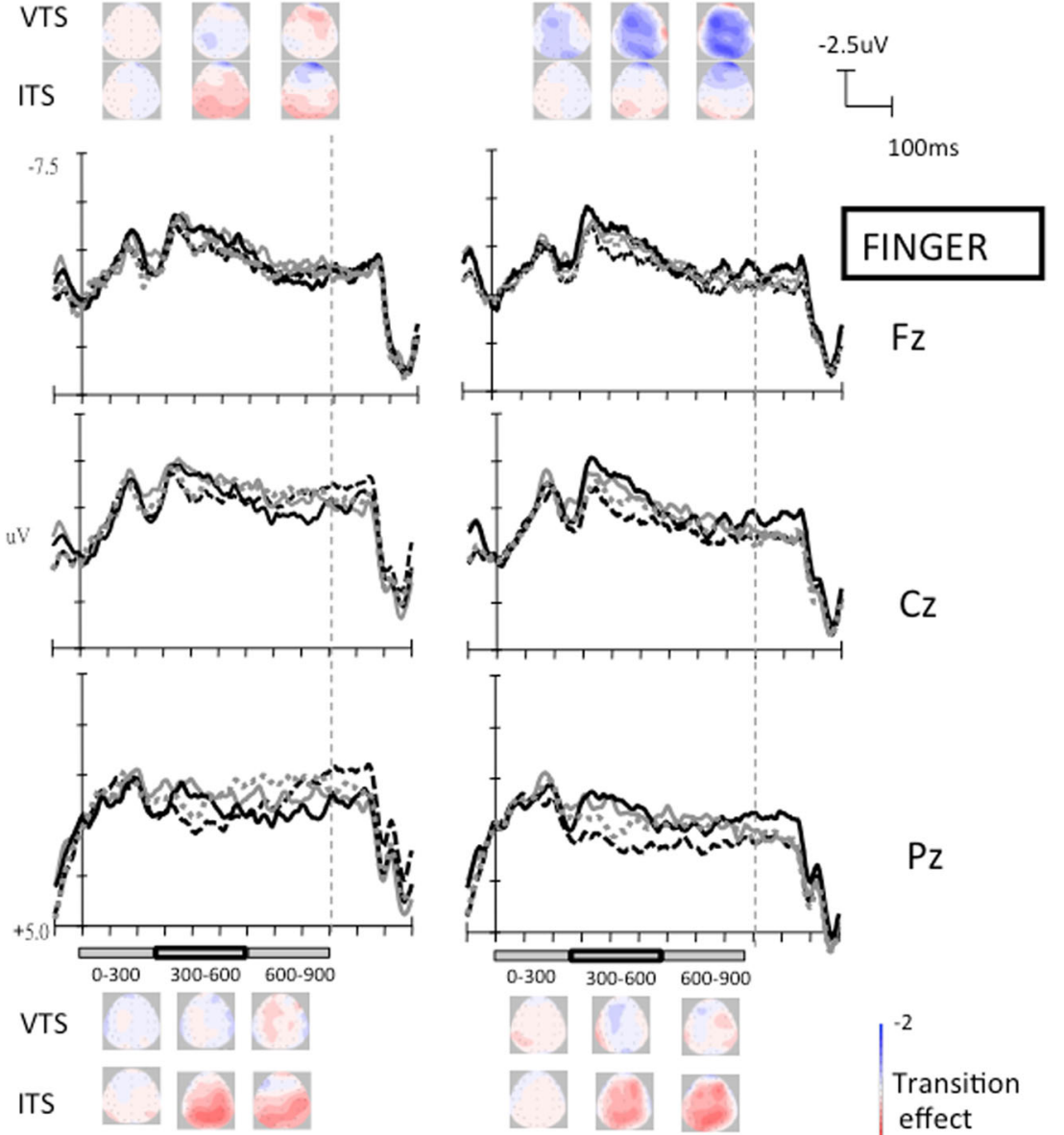

cueSwt/VTSswt cueRep/VTSrep

ITS switch ITS repeat

Fig. 3 Speed analysis of cue-locked event-related potential (ERP) waveforms for cue-target interval (CTI) $900 \mathrm{~ms}$. The left column represents the waveforms for fast trials while the right column represents the waveforms for slow trials. The topographic maps separately represent the transition main effects in three time windows for the voluntary switching (VTS) and instructed task switching (ITS), respectively. The vertical grey dashed line indicates the target onset 
The effectiveness index of preparation: fast-slow analysis

To simplify the presentation, we focused on the CTI 900-ms condition with separated fast-slow ERP modulations in the left- and right- column in Fig. 3. Given the complexity of the analysis, we decided to address the most salient effect as revealed in the previous analysis. That is, a four-way ANOVA (mapping $\times$ paradigm $\times$ transition $\times$ speed) was conducted at $\mathrm{Pz}$ for each 300-ms time window. The four-way interaction was significant in the $300-600-\mathrm{ms}$ time window, $F(1,46)=20.19$, $p<.01$.

The following simple interaction effects revealed a rather different speed modulation between HAND and FINGER groups in the VTS and ITS paradigm, respectively. Interestingly, the posterior negativity for switch trials (and/or posterior positivity for repeat trials) in the VTS paradigm was enhanced on slower trials, instead of fast trials, in the HAND group $(F(1,23)=7.06, p<.05)$, while no switch-related modulations were observed in the FINGER group regardless of fast or slow trials. For the switch-related posterior positivity in the ITS, the results replicated previous findings (Lavric et al., 2008) by showing an increased switch-related positivity on fast trials regardless of HAND $(F(1,23)=7.32, p<.05)$ or FINGER $(F(1,23)=31.38, p<.05)$ groups.

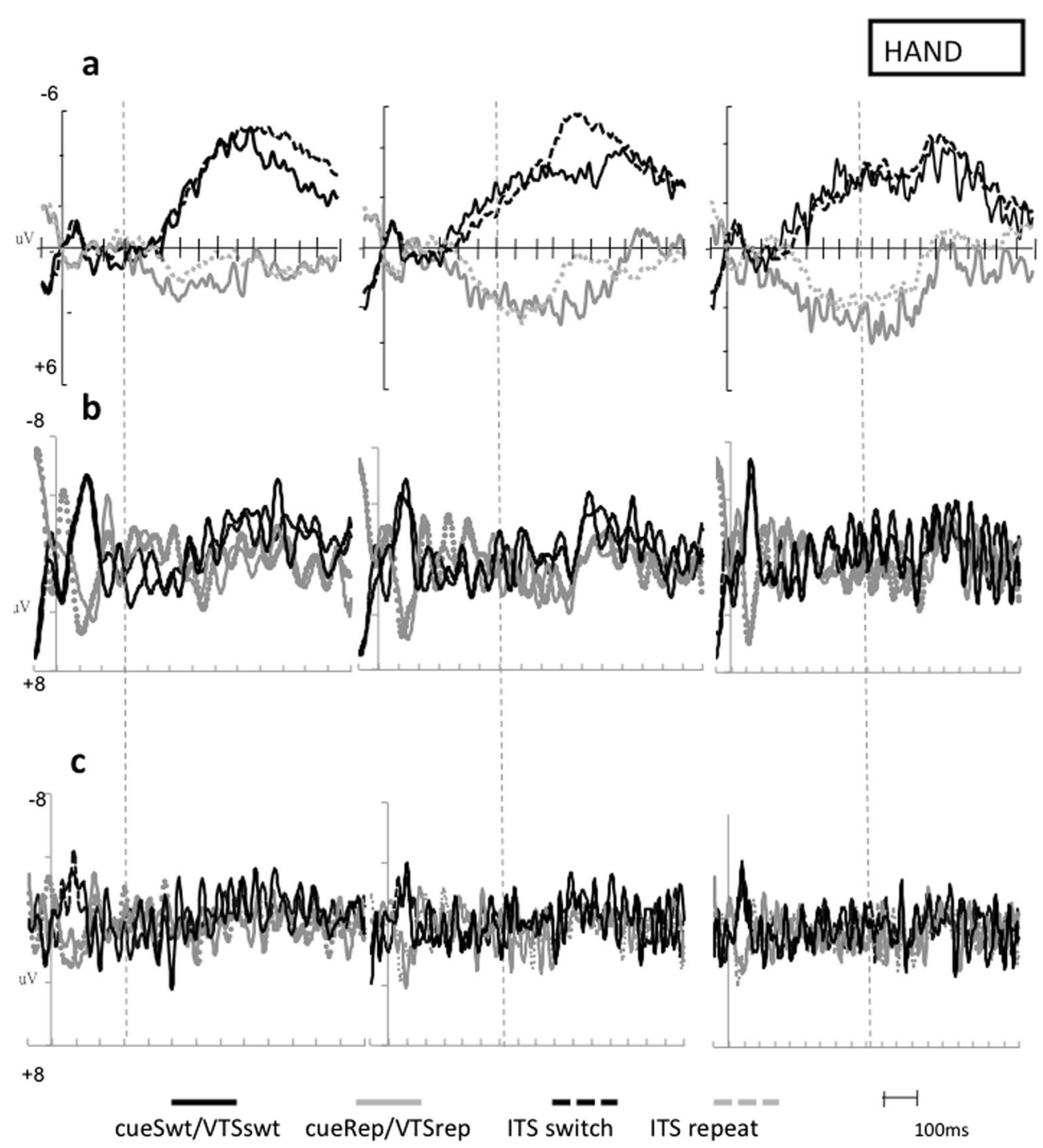

Fig. 4 The motor-specific preparation. The pretrial period lateralized readiness potential (LRP) (a) and the mu- (b) and beta- (c) movementrelated amplitude asymmetry (MRAA) are time-locked to the cue onset. Both the repeat and switch trials in the voluntary switching (VTS) and instructed task switching (ITS) paradigms are separated at different cuetarget interval (CTI) levels for the HAND group. The vertical grey dashed line indicates the target onset 
Hand-specific motor preparation analysis

Figure 4 represents the hand-specific motor preparation analysis, including the LRPs (4a) and the mu- (4b) and beta- (4c) MRAAs, for the three CTIs in the HAND group based on the selected task/hand on trial $n$. That is, the negative values correspond to the selection of the current response hand.

\section{$L R P S$}

Our data showed initial positive and negative dips for the repeat and switch trials (from 0-200 ms after cue onset), respectively, regardless of the VTS or ITS paradigms, and this might reflect the motor decay of the previous response given the short RCI. Following this "opposite" dip, the LRPs for the switch trials showed a negative-going deviation sustained up to target onset, but the LRPs for the repeat trials showed a positive-going deviation and did not revert to negative-going until $250 \mathrm{~ms}$ after target onset (see also Kang et al., 2014). Such findings of a positive-going deviation of the repeat trials before target onset seemed to be consistent with the view of a response-switch bias, in which the LRPs develop contrary to the n-1 response (Steinhauser, Hubner, \& Druey, 2009). Moreover, we did not observe a positive-going deflection (incorrect hand) of the LRPs for the switch trials before the target onset as revealed by Vandamme et al. (2010). Although the cause of the discrepancy between Vandamme et al. (2010) and the current findings is not clear, it might be related to the fundamental procedural difference (i.e., the cuing procedure) and the different baseline correction.

Nevertheless, the critical aspect of the LRP analysis was to clarify whether the hand-specific motor preparation was comparable between the VTS and ITS paradigms. As shown in Fig. 4, no significant difference between the VTS and ITS paradigms was identified in the switch and repeat trials, respectively, during the CTIs. These null effects appear to confirm that the motor component of translating the task goals into the response execution was identical between the VTS and ITS paradigms.

\section{mu- and beta-MRAAs}

Similar to the LRPs findings, the mu- (Fig. 4b) and beta(Fig. 4c) MRAAs showed initial positive and negative dips (0-200 ms after cue onset) for the repeat and switch trials, respectively, regardless of the VTS or ITS paradigm. Following the dips, it seemed that no particular consistent deflections were identified except for the mu and beta oscillations. With regard to the discrepancies between the current results and the study of Poljac and Yeung (2014), further studies are required.
Critically, the continuous t-test analysis for contrasting the VTS with ITS paradigms in the repeat and switch trials, respectively, did not reveal any successive difference during the CTIs.

\section{Discussion}

The present study clarified whether and how the effector-totask assignment modulated the advanced preparation between VTS and ITS. Of importance, we demonstrated the neural activities of the advanced preparatory processes in the VTS, but not in the ITS, were dependent on the effector-to-task assignment. This finding indicated that participants strategically prepared for the forthcoming task when they could voluntarily choose the task. Moreover, such an effectordependent preparatory strategy in the VTS was not the byproduct of the hand-specific motor preparation.

\section{Behavioral evidence of reconfiguration processes}

Consistent with previous findings (Arrington \& Logan, 2005), the current results showed increased repetition bias with insufficient preparation time in both effector-to-task mapping groups. As for the task performance (including RTs and PE), the reduction in switch costs (RISC) with advanced preparation in both the VTS and ITS paradigms demonstrated the involvement of endogenous control, which was independent of the effector-to-task assignment.

Even though neither the repetition bias nor the RISC effect from the ANOVAs showed effector-related differences, it is worth noting two planned comparisons for the effector-related facets: one is the overall repetition rate was lower for the FINGER group compared with the HAND group, while the other is the lengthening of RTs for the VTS paradigm as compared to the ITS paradigm in the FINGER group (see also Chen \& Hsieh, 2013). As we proposed the possibility of a different preparatory strategy, participants in the FINGER group did not make a task choice until the formation of an S-R correspondence effect as reflected by the inferior RTs of VTS as compared to ITS. Thus, the choice probability was further modulated by the S-R correspondence effect in addition to the representativeness and availability heuristic (for details, see Arrington \& Logan, 2005). Given the role of the S$\mathrm{R}$ correspondence effect on choice probability, the availability heuristic for repeating the same task would be reduced as reflected by the lower repetition bias in the FINGER group as compared to the HAND group. Taken together, the behavioral effector-related effects were in line with speculations that the effector manipulation led participants to adopt diverse preparatory strategies. 
Neural evidence of differential reconfiguration processes between VTS and ITS

The general findings indicated that the neural mechanism underlying the preparatory processes of the VTS was quite different to that underlying the ITS (Fig. 2). Of importance, the present study revealed that the effector manipulation contributed to a differential preparatory strategy in the VTS context, but not in the ITS context. Thus, we divided the discussion into effector-dependent effect in the VTS and effector-independent effect in the ITS, respectively. A final remark would further address the general effector effect.

\section{The effector-to-task mapping dependent preparatory strategy in VTS}

Of interest, the current results extended previous findings to reveal the effector-dependent switch-related modulations when participants could make their own decisions. Under the VTS context, participants in the HAND group showed increased negativities for voluntarily switching while participants in the FINGER group did not reveal any switch-related modulations. Accordingly, it seemed reasonable to link the ERP modulation in the HAND group to increased switch-related negativities under the construct of endogenous control, i.e. the involvement of intentional control (Poljac \& Yeung, 2014) or existence of a reconfiguration process (Vandamme et al., 2010). However, the speed ERP analysis revealed another facet for re-evaluating endogenous control in the VTS context. That is, the results demonstrated that increased switch-related negativities were observed in the slow trials, instead of the fast trials (Fig. 3), which was contrary to the argument of effective preparation for reconfiguration in the ITS (see Lavric et al., 2008). Thus, we proposed an alternative construct by inferring that the ERP modulations are the repeat-related positivity. According to the inhibitory strategy in the VTS (Mayr \& Bell, 2006), the slower repeat RTs represented greater retrieval demand of the most recently "inhibited" repeating trials, which seemed to be in line with the repeat-related positivity on slow trials. Although this argument was contrary to the core concept of reconfiguration in the ITS, we think this discrepancy might reflect that the underlying mechanism of task switching was different between VTS and ITS (see also Kang et al., 2014). Further studies are required to address this issue.

However, the null switch-related effect in the FINGER group seemed to suggest no switch-specific preparation occurred before target presentation. With the speed ERP analyses discarding the preparatory jitter-in-time possibility (see Fig. 3), the most plausible explanation was participants strategically prepare the upcoming stimulus by keeping both tasks available and make a task choice at target onset (for a similar argument in the ITS, see Hsieh \& Chen, 2006). With regard to the possible mechanism for general preparation, we speculated the possibility of a contribution of bindings between the digit stimuli and the effectorto-finger assignment (Chen and Hsieh, 2013). That is, common code representation led participants not to prepare specific tasks before target onset so that null ERP modulations were observed. Nevertheless, one has to be cautious about claiming general preparation as one of the VTS preparatory strategies since it might not be valid in the context of other stimuli.

Overall, the most valuable implication we raised was participants were able to generate different strategies based on their own will. In the present study, we revealed the role of effectordependent preparation in the VTS context even though the instruction was identical between the two effector-to-task groups. The present results raised doubts about the existence of a common/unified endogenous control in the VTS context (see also Demanet \& Liefooghe, 2013). Furthermore, the present study revealed the benefit of adopting ERP measurements to dissociate the differential preparatory strategy through sensitive neural measurements that might not be detected through behavioral measurements. As noted by Demanet and Liefooghe (2013), the behavioral RISC effect was unable to catch the topdown component in the VTS context.

\section{The effector-to-task mapping independent preparatory strategy in ITS}

Contrary to the effector-dependent ERP modulations in the VTS context, the late posterior positivity that began at approximately $300 \mathrm{~ms}$ and extended to $600-700 \mathrm{~ms}$ was larger for the switch trials than for the repeat trials in the ITS paradigm regardless of the HAND or FINGER group (see Fig. 2). Notably, the effector-independent preparation property appears to suggest a common reconfiguration process in ITS (Hsieh \& Wu, 2011). Once the specific task goal is formed by the instructions (i.e., the cue indicates which task to perform), the effector-to-mapping assignment served as the bridge between the stimulus and the response.

Nevertheless, one might argue that the posterior switchrelated positivity was confounded with the cue transition effect with regard to the 1:1 cue-to-task mapping (Logan \& Bundesen, 2003). Although the current design was unable to exclude this possibility, previous studies have indicated that the posterior switch-related positivity was associated with the task switch effect more than the cue transition effect (Hsieh \& $\mathrm{Wu}, 2011$; Jost et al., 2008). Of importance, we believe that the differential ERP modulations between the VTS and ITS in the present study were not simply the byproduct of the cue transition effect with respect to the equal cue/task transition comparison between VTS and ITS. 


\section{The effector effect on overall performance}

In addition to the switch-specific effect, it was also interesting to understand whether the effector manipulation altered the overall task performance, i.e., the distinct hand-to-task association might be more beneficial to maintain the S-R rule as compared to finger-to-task association. Clearly, the overall increased posterior negativity of the HAND group as compared to the FINGER group, regardless of VTS or ITS, suggested that the hand-to-task association provides more efficient S-R maintenance (Verleger et al., 2000).

\section{The role of hand-specific motor preparation}

Furthermore, given the concurrent property between task switching and hand shifting in the HAND group, the foreperiod of the LRPs and the mu- and beta-MRAA served as an objective index for differentiating the hand-specific motor preparation component from non-motor preparation. Of importance, none of these components showed a significant difference between VTS and ITS for the switch trials or repeat trials, which suggests that the motor-specific preparation of VTS was identical to ITS. Thus, it is difficult to reconcile that the observed posterior ERP modulations of the HAND group in the VTS context was merely due to hand shifting.

\section{Conclusions}

Collectively, the current data provide the neural evidence to show a differential preparatory process between VTS and ITS paradigms. Because of the fundamental aspect of "will," the present study revealed that a preparatory strategy could be further modulated by the effector-to-task assignment in the VTS context. However, the preparatory process in the ITS paradigm was insensitive to the effector-to-task assignment manipulation because the recruitment of reconfiguration was correlated with an external instruction rather than "voluntary will."

Acknowledgments The authors would like to thank the Ministry of Science Technology (original: National Science Council) of the Republic of China, Taiwan for financially supporting this research (Contract No.NSC98-2410-H-006-113-MY3).

\section{References}

Allport, A., Styles, E. A., \& Hsieh, S. (1994). Shifting intentional set: Exploring the dynamic control of tasks. In C. Umilta \& M. Moscovitch (Eds.), Attention and performance XV (pp. 421-452). Hillsdale, NJ: Lawrence Erlbaum Accociate, Inc.
Arrington, C. M. (2008). The effect of stimulus availability on task choice in voluntary task switching. Memory \& Cognition, 36(5), 991-997.

Arrington, C. M., \& Logan, G. D. (2004). The cost of a voluntary task switch. Psychological Science, 15(9), 610-615.

Arrington, C. M., \& Logan, G. D. (2005). Voluntary task switching: chasing the elusive homunculus. Journal of Experimental Psychology: Learning, Memory, and Cognition, 31(4), 683-702.

Astle, D. E., Jackson, G. M., \& Swainson, R. (2008). Fractionating the cognitive control required to bring about a change in task: a densesensor event-related potential study. Journal of Cognitive Neuroscience, 20(2), 255-267.

Barcelo, F., Munoz-Cespedes, J. M., Pozo, M. A., \& Rubia, F. J. (2000). Attentional set shifting modulates the target $\mathrm{P} 3 \mathrm{~b}$ response in the Wisconsin card sorting test. Neuropsychologia, 38(10), 1342-1355.

Barcelo, F., Perianez, J. A., \& Knight, R. T. (2002). Think differently: a brain orienting response to task novelty. Neuroreport, 13(15), 1887-1892.

Chen, P., \& Hsieh, S. (2013). When the voluntary mind meets the irresistible event: Stimulus-response correspondence effect on the task selection during voluntary task switching. Psychonomic Bulletin \& Review, 20(6), 1195-1205.

Coles, M. G. (1989). Modern mind-brain reading: psychophysiology, physiology, and cognition. Psychophysiology, 26(3), 251-269.

de Jong, R., Gladwin, T. E., \& 't Hart, B. M. (2006). Movement-related EEG indices of preparation in task switching and motor control. Brain Research, 1105(1), 73-82.

Demanet J., \& Liefooghe B. (2013). Component processes in voluntary task switching. Quarterly journal of experimental psychology

Donchin, E., \& Coles, M. G. H. (1988). Is P300 component a manifestation of context updating? Behavioral and Brain Sciences, 11, 357-427.

Hsieh, S., \& Chen, P. (2006). Task reconfiguration and carryover in task switching: an event-related potential study. Brain Research, 1084(1), 132-145.

Hsieh, S., \& Wu, M. (2011). Electrophysiological correlates of preparation and implementation for different types of task shifts. Brain Research, 1423, 41-52.

Jost, K., Mayr, U., \& Rosler, F. (2008). Is task switching nothing but cue priming? Evidence from ERPs. Cognitive, Affective, \& Behavioral Neuroscience, 8(1), 74-84.

Kang, M. S., Diraddo, A., Logan, G. D., \& Woodman, G. F. (2014). Electrophysiological evidence for preparatory reconfiguration before voluntary task switches but not cued task switches. Psychonomic Bulletin \& Review, 21(2), 454-461.

Karayanidis, F., Coltheart, M., Michie, P. T., \& Murphy, K. (2003). Electrophysiological correlates of anticipatory and poststimulus components of task switching. Psychophysiology, 40(3), 329-348.

Karayanidis, F., Jamadar, S., Ruge, H., Phillips, N., Heathcote, A., \& Forstmann, B. U. (2010). Advance preparation in task-switching: converging evidence from behavioral, brain activation, and modelbased approaches. Frontiers in Psychology, 1, 25.

Kieffaber, P. D., \& Hetrick, W. P. (2005). Event-related potential correlates of task switching and switch costs. Psychophysiology, 42(1), $56-71$.

Kiesel, A., Steinhauser, M., Wendt, M., Falkenstein, M., Jost, K., Philipp, A. M., \& Koch, I. (2010). Control and interference in task switching-a review. Psychological Bulletin, 136(5), 849-874.

Lavric, A., Mizon, G. A., \& Monsell, S. (2008). Neurophysiological signature of effective anticipatory task-set control: a task-switching investigation. European Journal of Neuroscience, 28(5), 1016-1029.

Liefooghe, B., Demanet, J., \& Vandierendonck, A. (2010). Persisting activation in voluntary task switching: it all depends on the instructions. Psychonomic Bulletin \& Review, 17(3), 381-386.

Logan, G. D., \& Bundesen, C. (2003). Clever homunculus: is there an endogenous act of control in the explicit task-cuing procedure? 
Journal of Experimental Psychology: Human Perception and Performance, 29(3), 575-599.

Mayr, U., \& Bell, T. (2006). On how to be unpredictable: evidence from the voluntary task-switching paradigm. Psychological Science, 17(9), 774-780.

Mueller, S. C., Swainson, R., \& Jackson, G. M. (2007). Behavioural and neurophysiological correlates of bivalent and univalent responses during task switching. Brain Research, 1157, 56-65.

Poljac, E., \& Yeung, N. (2014). Dissociable Neural Correlates of Intention and Action Preparation in Voluntary Task Switching. Cerebral Cortex, 24(2), 465-478.

Rogers, R. D., \& Monsell, S. (1995). Costs of a predictable switching between simple cognitive tasks. Journal of Experimental Psychology: General, 124(2), 207-231.
Steinhauser, M., Hubner, R., \& Druey, M. (2009). Adaptive control of response preparedness in task switching. Neuropsychologia, 47(89), 1826-1835.

Van Boxtel, G. J. M., \& Böcker, K. B. E. (2004). Cortical Measures of Anticipation. Journal of Psychophysiology, 18(2-3), 61-76.

Vandamme, K., Szmalec, A., Liefooghe, B., \& Vandierendonck, A. (2010). Are voluntary switches corrected repetitions? Psychophysiology, 47, $1176-1181$.

Vandierendonck, A., Liefooghe, B., \& Verbruggen, F. (2010). Task switching: interplay of reconfiguration and interference control. Psychological Bulletin, 136(4), 601-626.

Verleger, R., Wauschkuhna, B., van der Lubbea, R., Jaśkowskia, P., \& Trillenberg, P. (2000). Posterior and anterior contribution of handmovement preparation to late CNV. Journal of Psychophysiology, 14(2), 69-86. 